\title{
Colliding winds in Wolf-Rayet binaries: further developments within a complicated story
}

\author{
Rolf Walder and Doris Folini \\ Institut für Astronomie, ETH Zürich, Switzerland \\ Simin M. Motamen \\ Seminar für Angewandte Mathematik, ETH Zürich, Switzerland
}

\begin{abstract}
We present large scale 3D simulations of colliding winds in the WR binary $\gamma^{2}$ Velorum (WR 11). The O-star wind is confined by cold, high density shells and forms a spirally shaped region within the WR-wind. As a consequence of the elliptic orbit, the opening angle and the curvature of the spiral as well as the ratio of the volumes occupied by the WR- and the $\mathrm{O}$-wind are functions of the orbital phase. Our model qualitatively reproduces the observed X-ray lightcurve. The impact of magnetic fields and heat conduction on the physics of colliding winds is briefly discussed and some remarks on the important question of stability are made ${ }^{1}$.
\end{abstract}

\section{Introduction}

Since the last Wolf-Rayet IAU symposium devoted to colliding winds (van der Hucht \& Williams 1995), many new results within this field have been achieved. Among the most exciting ones are the possible radiative braking of the WRwind by the radiation field of the O-star (Gayley, Owocki \& Cranmer 1997) and the first radio images of the wind collision zone (e.g., Williams; Dougherty \& Williams; Watson et al.; Contreras \& Rodríguez, all in these Proceedings).

In this paper we present 3D, large scale simulations of $\gamma^{2}$ Vel which can reproduce the observed X-ray light-curve of this system (Section 2). In Section 3 we discuss some issues related to the stability properties of colliding winds and in Section 4 the possible impact of magnetic fields and heat conduction on the physics of colliding winds. In Section 5 we draw conclusions. All simulations presented within this paper are computed by our AMRCART-code which can be obtained for free by anybody.

\section{2. $\gamma^{2}$ Velorum: X-ray light-curve and structure of the circumstellar matter}

In this section we present simulations of the WR binary $\gamma^{2}$ Vel (WR 11). However, many statements qualitatively also apply to other WR binaries. For our simulation we have chosen system parameters adopted from Schmutz et al.

\footnotetext{
${ }^{1}$ Papers by the authors, colored versions of the pictures, and movies can be copied from $<$ http://www.astro.phys.ethz.ch/staff/walder/walder.html >. The AMRCART-code can be ordered from <walder@astro.phys.ethz.ch>.
} 
(1997): period $P=78.53 \mathrm{~d}$, eccentricity $\epsilon=0.326$, inclination $i=65^{\circ}$, a periastron angle of the WR-star of $68^{\circ}, 9 \mathrm{M}_{\odot}$ for the WR star and $29 \mathrm{M}_{\odot}$ for the O-type star. From Schaerer, Schmutz \& Grenon (1997) we take $v_{\infty}=1450 \mathrm{~km} \mathrm{~s}^{-1}$ for the WR wind. With $10^{-5} \mathrm{M}_{\odot \mathrm{y}^{-1}}$, we choose a lower value for the mass loss rate than suggested by these authors to account for a clumpy wind. For the O-star wind, we adopt $4 \times 10^{-7} \mathrm{M}_{\odot} \mathrm{y}^{-1}$ and $v_{\infty}=2300 \mathrm{~km} \mathrm{~s}^{-1}$ (W. Schmutz, private communication). We set the temperature of the undisturbed winds to $15000 \mathrm{~K}$. We do not consider radiative braking and use the terminal velocity of the winds straight from the stars. We also neglect radiative cooling in this simulation.

\subsection{Shocks, spirals, and the confinement of the O-star wind}

The X-ray photons are exclusively produced in the inner region of the binary. The shape of the light-curve, however, is essentially determined by the large scale structure of the circumstellar matter. Since the WR-wind is opaque, the $\mathrm{X}$-ray photons can only escape through the less dense O-star wind (Stevens, Blondin \& Pollock 1992; Willis, Schild \& Stevens 1995; Stevens et al. 1996).

Whereas the structure in the region between the two stars is dominated by the two strong shocks where the two winds crash together (Figure 1), the large scale circumstellar structure is dominated by the spirally distributed $\mathrm{O}$ star wind, embedded in the WR-wind (Figure 2).

First, we focus on the central region (Figure 1). To first approximation, this region is nearly axisymmetric and has characteristics as described by Stevens, Blondin \& Pollock (1992). We note that the shocks are strong only in the very central region, where the flow hits them head on. In a plane through the O-star perpendicular to the axis connecting the two stars, the shocks have already significantly weakened and beyond this plane they finally fade away nearly completely. However, the interaction region is still significantly hot, due
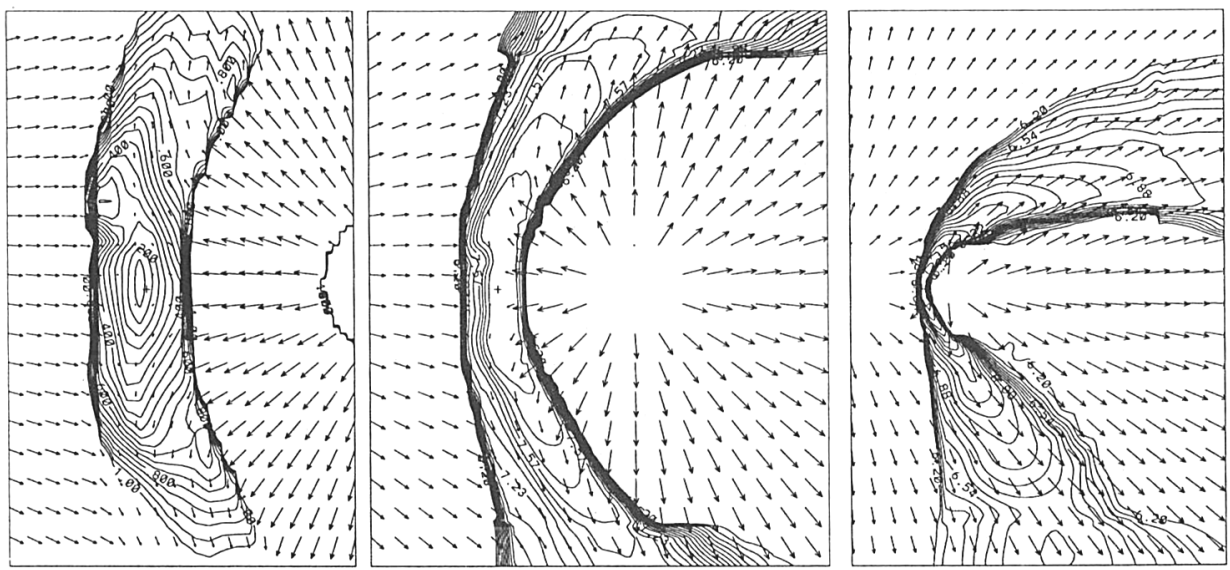

Figure 1. $\quad \gamma^{2}$ Vel: slices in the orbital plane at periastron showing subsequently larger areas. The stars orbit counterclockwise. The O-star, visible on the right of the left panel, has a radius of $13 R_{\odot}$. Shown is the projected velocity field (largest vector $v_{\infty_{0}}=2450 \mathrm{~km} \mathrm{~s}^{-1}$ ), together with $20 \mathrm{Mach}$ number contours between 9 and 1 (left), and 20 temperature contours between $10^{6} \mathrm{~K} \leq T \leq 10^{8} \mathrm{~K}$ (middle and right). 

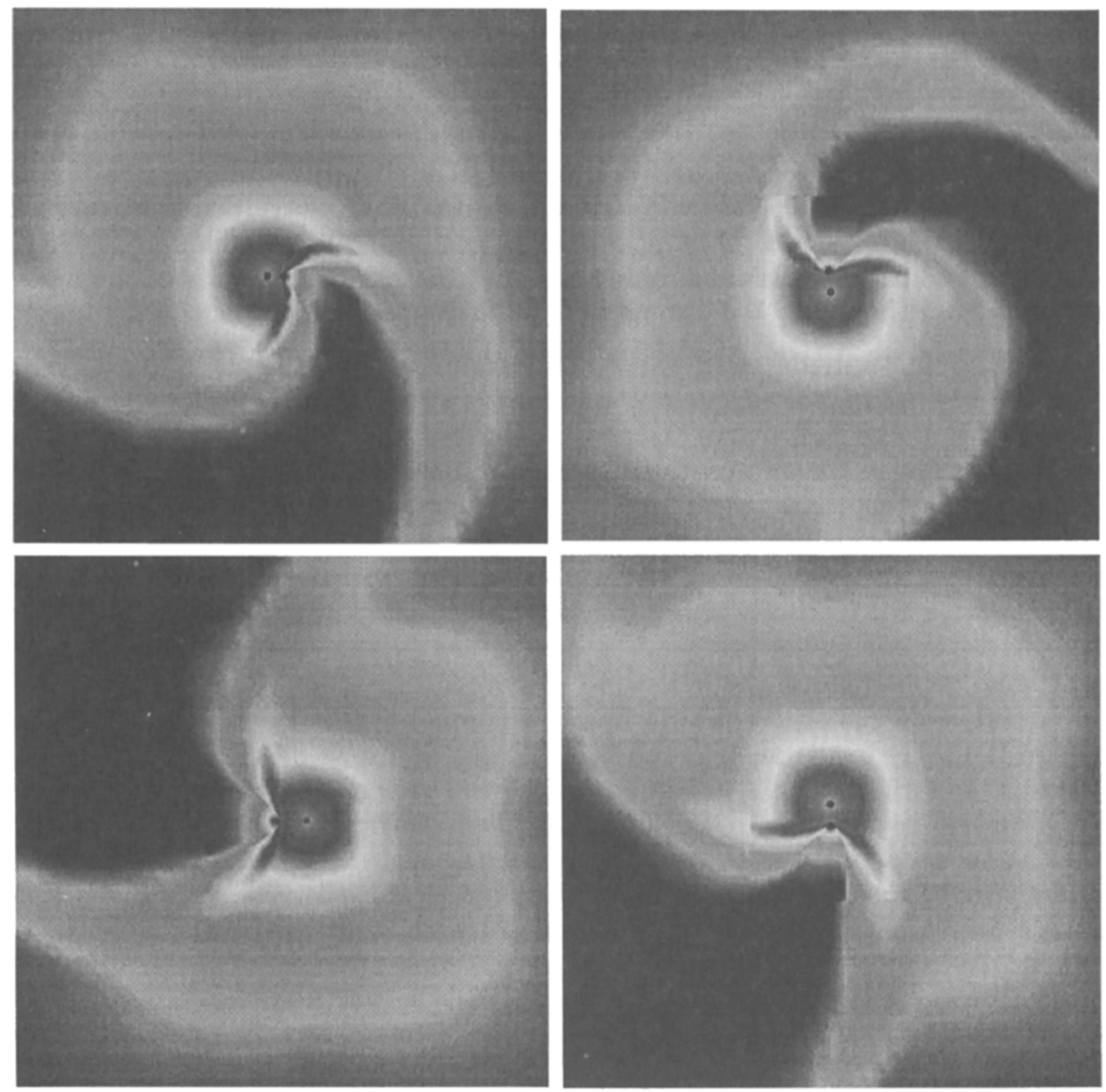

Figure 2. $\quad \gamma^{2}$ Vel: slices in the orbital plane at periastron (upper left), in the first quarter (upper right), in apastron (lower left), and in the third quarter (lower right). The stars, shown (enlarged) as black dots, orbit counterclockwise. Shown is density in grey scale: dark(high density)-white-grey(low density). The O-star wind is shown in black (except for the central region).

to the transport of hot gas from the central region. Proceeding outwards, the influence of the orbital motion steadily increases and the spirally shaped large scale structure grows.

The large scale structure is dominated by the spirally shaped pattern of the $\mathrm{O}$-star wind, confined within the WR-wind (Figure 2). In circularized systems the pattern is stationary in co-rotating coordinates. Here, with an elliptic orbit, the volume occupied by the $\mathrm{O}$-star wind as well as the opening angle and the curvature of the spiral is varying with the orbit. Although the pattern rotates with the orbit, the matter does not. Outside of the central region the particles of both winds essentially move radially outwards. As described above, the shock structure in the central region lets escape only a part of the O-star wind. On the 
large scale, the location of the O-star wind is determined by the outward motion of the escaped particles relative to the orbital motion. Looking at the waves forming the interaction region and confining the O-star material, there is a clear difference between the leading waves pushing the structure (weak shocks) and the trailing edge of the structure (rarefaction waves). The large scale structure strongly depends on the ratio between wind speeds and the orbital speeds of the stars (Walder 1999).

Favorable temperatures for $X$-ray emission lie above some $10^{7} \mathrm{~K}$ (Bremsstrahlung) and below a few times $10^{6} \mathrm{~K}$ (line emission). In $\gamma^{2}$ Vel (Figure 1 ) such temperature ranges are found in the very central region and then again slightly further away from the center. The exact position of the outer region is given by the ratio of the cooling time to the transport time. We emphasize that adiabatic cooling can essentially contribute if, for temperatures between a few $10^{6}$ to $10^{7} \mathrm{~K}$, radiative cooling is ineffective. Due to the nearly cylindrical symmetry adiabatic cooling is approximately proportional to the distance from the axis connecting the two stars.

\subsection{X-ray light-curve}

Based on this first 3D simulation of $\gamma^{2}$ Vel we have computed the shape of the $\mathrm{X}$-ray light-curve under the simplified assumption of constant opacity through the WR wind. We have taken four different points in the center of the system and have computed the column density along the rays to the observer. Figure 3 shows the flux an observer would measure if these four points would have the same emission. This very simple model gives a surprisingly good qualitative agreement with the measured X-ray light-curve by Willis, Schild and Stevens (1995). In particular, the phases of the peak emission correspond very well. Both curves have a very sharp increase towards the maximum and a more flat decrease, the model decreasing a little more flat than the observation. The model also shows the little plateau before the emission completely vanishes around phase 0.8. Even though this model is clearly limited it shows that the X-ray light-curve of a WR binary is mostly determined by the large scale structure of the circumstellar matter. Figure 4 illustrates the influence of inclination and periastron angle on the X-ray light-curve.
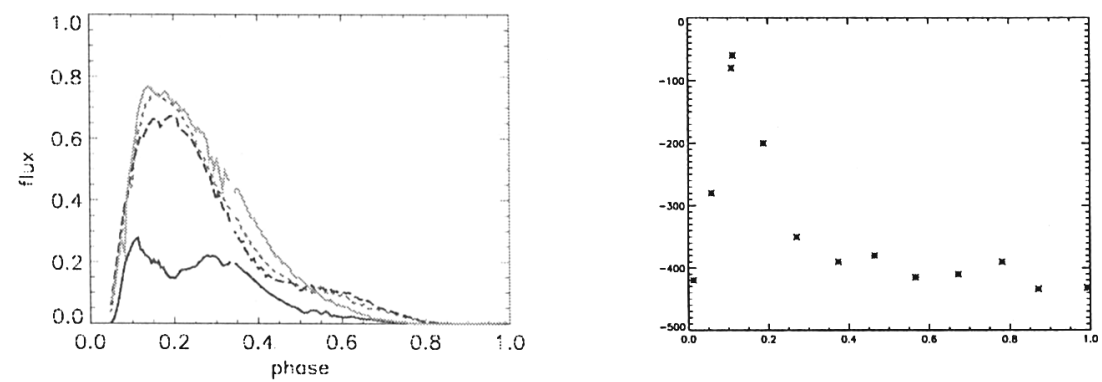

Figure 3. Computed light-curves from four different emission points (left), and observed light-curve by Willis, Schild \& Stevens (1995) (right). Note that phase zero coincides with the periastron. 

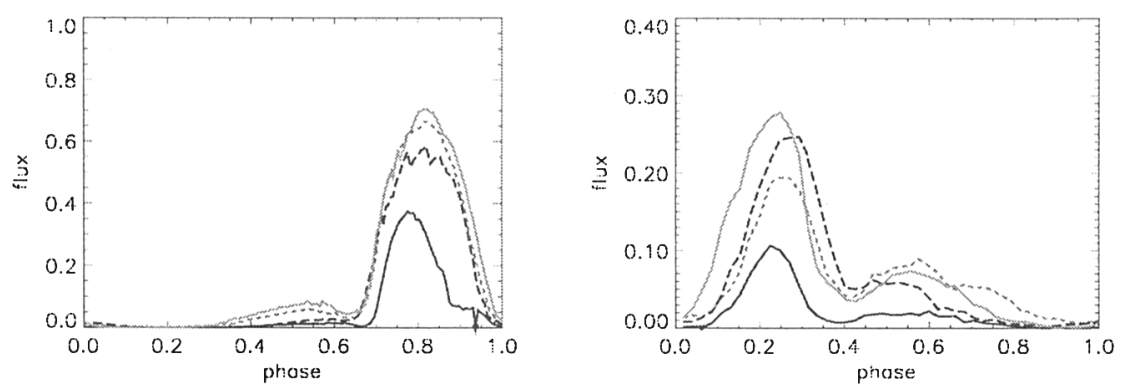

Figure 4. X-ray light-curve if $\gamma^{2}$ Vel had inclination $i=65^{\circ}$, periastron angle $\omega_{\mathrm{WR}}=248^{\circ}$ (left), and if inclination $i=30^{\circ}$, periastron angle $\omega_{\mathrm{WR}}=90^{\circ}$ (right).

\subsection{What about the cold part of the interaction zone?}

The shocked material, after cooling by X-ray emission, is strongly compressed into thin layers which confine the O-star wind. Numerical simulations show (see Section 3 ) that these layers are most likely unstable and in a state of supersonic turbulence, leading to a large velocity and density dispersion.

On the orbital scale, due to their high density, these layers can contribute to the optical and UV spectrum of the system (see also Hill et al.; Moffat, all in these Proceedings). However, their temperature, ionization, and excitation state are difficult to compute since they are essentially determined by the radiation fields of the stars, the absorption of the X-rays of the shocked matter, and by fast electrons originating from shocked matter. Due to the high densities the influence of the stellar radiation has to be treated by 3D optically thick NLTE radiative transfer. In her thesis Folini (1998) has developed a code which is able to fulfill this task (see Folini \& Walder 1999 for a concise version). As a first application, we have computed the ionization state of the interaction zone for a toy example. The results can be taken from Folini \& Walder (these Proceedings).

On a larger scale, these layers seem to favor dust production. Surprisingly, in many WR-binaries dust is observed even at distances so close to the stars that its production should be suppressed by the still strong radiation field (see e.g., Williams, these proceedings). Supersonic turbulence may be a mechanism to overcome this problem as it can generate very compressed spots which possibly lead to conditions under which dust can form.

\section{The crucial issues of stability}

A wide range of numerical simulations show that the interaction zone is unstable whenever the shocks are radiative. There is, however, no consistent picture since different codes give different results, from wildly unstable to nearly stable.

There are several reasons why the question of stability is crucial from an astronomical point of view, apart from the fundamental desire to understand the physics involved. X-ray observations (e.g., Stevens et al. 1996) indicate variations on small time scales. Instabilities would produce hot regions of various temperatures, densities and extensions, all variable in time. The resulting 
effects on the X-ray emission may become measurable with a new generation of satellites. Instabilities also would likely lead to supersonic turbulence within the highly compressed cold part of the interaction zone (see e.g., Walder \& Folini 1998), leading to a large velocity- and density dispersion, visible in the emitted spectrum. The production of dust associated with the interaction zone may be affected by such instabilities. Similar instabilities and supersonic turbulence are possibly involved in triggered star formation in starburst regions (Hunter et al. 1986; Mac Low et al. 1998).

We have to admit that hardly anything is understood about instabilities of colliding winds in massive binaries. (A brief review of many aspects of the stability of radiative flows can be found in Walder \& Folini 1999a.) In most simulations of real systems the very central region appears to be stable and dynamical instabilities are excited only relatively far away from the central axis (e.g., the V444 Cyg simulation by Stevens, Blondin \& Pollock 1992 or Walder $\&$ Folini 1995, the O+O star simulations by Pittard \& Stevens 1997). The same is observed in the simulations by Myasnikov, Zhekov \& Belov (1998) who, in addition, find that the growth rate of the instabilities depends strongly on the temperature of the cold zone. How the instability in these outer regions is excited is not clear. It cannot be explained by the mechanism described by Vishniac (1994). While this mechanism works in planar symmetry - before it saturates into supersonic turbulence (Blondin \& Marks 1996; Walder \& Folini $1999 \mathrm{~b})$ - it can be ruled out here as there is no substantial ram-pressure on the cold sheet in the outer regions. The model by Dgani, Walder \& Nussbaumer (1993) may provide a more suitable explanation, but further investigations are necessary to clear this point.

Another important fact Myasnikov, Zhekov \& Belov (1998) pointed out is the singularity of the stagnation point. While it seems not clear whether there exists at all a stable configuration of the stagnation point in the presence of orbital motion, the point they make is important in a more general context. As Figure 1, left, illustrates the flow in the center-most region of the interaction zone is very subsonic. The material may, therefore, cool before being transported away. In numerical simulations this very subsonic transport depends strongly on the numerical grid. On relatively coarse grids the material is transported away too fast. On the other hand, high-resolution simulations by Walder \& Folini (1995) show indeed cold condensations at the very center.

Finally, we would like to stress that the above picture needs revision in the case of clumpy winds. Clumpiness would provide density variations within the interaction zone, which are able to powerfully excite a whole variety of instabilities. Results from numerical simulations in the pioneering work by Owocki, Castor \& Rybicki (1988), the analysis of the time evolution of substructure in observed spectra (Lépine, Moffat \& Henriksen 1996) as well as the comparison of model WR-atmospheres with observations (see Hamann et al.; Hillier; and Schmutz \& De Marco, all in these Proceedings) indeed indicate clumpy winds.

\section{Magnetic fields and heat conduction}

Magnetic fields There are several good reasons to seriously attack the question of the possible presence of magnetic fields in WR-binaries. The most prominent is perhaps the unambiguously observed non-thermal emission (van 
der Hucht 1992; Williams; Contreras \& Rodríguez; Dougherty; Watson et al., all in these Proceedings) from many of these binaries. All emission mechanisms proposed so far need the presence of magnetic fields of at least some Gauss, many of them need several hundred Gauss. Estimates show that field strengths of this order may influence the dynamics of at least the O/B-star winds and certainly will contribute to the shape and the stability properties of the wind-wind interaction zone. First numerical experiments done by our group confirm this. Further, we point to an interesting proposition by Gonçalves, Jatenco-Pereira \& Opher (1998), who assign wind-inhomogeneities in WR-atmospheres to Alvén waves. Finally, heat transfer by thermal electrons - an otherwise very powerful process - is nearly completely suppressed normal to the magnetic field.

Heat conduction Heat from zones of shocked gas can efficiently be transfered by thermal electrons to neighboring regions. Motamen, Walder \& Folini (these Proceedings) show that in the absence of magnetic fields, this process considerably softens the X-ray emission. The spectrum contains more emission lines and becomes a composite of the emission from high- and low density regions.

\section{Conclusions}

By means of $3 \mathrm{D}$ numerical simulations we have shown the differences and the connection between the central shock structure and the spirally shaped circumstellar matter in colliding wind WR binaries.

On this basis the enhanced X-ray production and the shape of the X-ray light-curve of WR binaries can be understood. In particular we have shown the good qualitative agreement between the observed and the computed light-curve in $\gamma^{2}$ Vel. Many details of the X-ray spectrum probably cannot be understood in such a simple way. This mostly concerns the possible impact of thermal heat transfer and of the variable emission due to unstable shocks. It will be a great challenge for the theoretical modeling and the numerical simulation to achieve progress in these topics. On the observational side, the next generation satellites will allow to study shock physics in much more detail.

We have emphasized the importance of studying the cold parts of the interaction zone. They probably contribute to the optical and UV spectrum of WR binaries and can favor dust production. We have developed a 3D optically thick NLTE code which will be able to compute this contribution. An open question remains how the compressed gas triggers dust production.

Acknowledgments. We thank Jean Favre for his support to visualize our data. We are grateful to Bruno Loepfe and all members of the supercomputer crew in Zürich and to Peter Steiner which maintains the system at our institute. We thank Werner Schmutz for fruitful discussions on Wolf-Rayet stars.

\section{References}

Blondin, J.M., Marks B.S. 1996, New Astronomy 1, 235

Dgani, R., Walder, R., Nussbaumer, H. 1993, A\&A 267, 155

Folini, D. 1998, ETH-Thesis, No. 12606.

Folini, D., Walder, R. 1999, in: Proc. 7th Intern. Conf. on Hyperbolic Problems (Basel: Birkhäuser), in press 
Gayley, K.G., Owocki, S.P., Cranmer, S.R. 1997, ApJ 475, 786

Gonçalves, D.R., Jatenco-Pereira, V., Opher, R. 1998, ApJ 501, 797

van der Hucht, K.A. 1992, The A\&A Review 4, 123

van der Hucht, K.A., Williams, P.M. (eds.) 1995, Wolf-Rayet Stars: Binaries, Colliding

Winds, Evolution, Proc. IAU Symp. No. 163 (Dordrecht: Kluwer)

Hunter, J.H., Sandford, M.T., Whitaker, R.W., Klein, R.I. 1986, ApJ 305, 309

Lépine, S., Moffat, A.F.J., Henriksen, R.N. 1996, ApJ 466, 392

Mac Low, M.-M., Klessen, R.S., Burkert, A., Smith, M.D. 1998, Phys. Rev. Letters 80, 2754

Myasnikov, A.V., Zhekov, S.A., Belov, N.A. 1998, MNRAS 298, 1021

Owocki, S.P., Castor, J.I., Rybicki, G.B. 1988, ApJ 335, 914

Pittard, J.M., Stevens, I.R. 1997, MNRAS 292, 29

Schaerer, D., Schmutz, W., Grenon, M. 1997, ApJ 484, L153

Schmutz, W., Schweickhardt, J., Stahl, O. et al. A\&A 328, 219

Stevens, I.R., Blondin, J.M., Pollock, A.M.T. 1992, ApJ 386, 265

Stevens, I.R., Corcoran, M.F., Willis, A.J., Skinner S.L., Pollock, A.M.T., Nagase, F., Koyama, K. 1996, MNRAS 283, 589

Vishniac, E.T. 1994, ApJ 428, 186

Walder, R., Folini D. 1995, in: K.A. van der Hucht \& P.M. Williams (eds.), WolfRayet Stars: Binaries, Colliding Winds, Evolution, Proc. IAU Symp. No. 163 (Dordrecht: Kluwer), p. 525

Walder, R., Folini, D. 1998, A\&A 330, L21

Walder, R. 1999, in: Hypersonic Radiative Outflows out of Thermal Equilibrium, ApSS in press

Walder, R. \& Folini, D. 1999a, in Hypersonic Radiative Outflows out of Thermal Equilibrium, ApSS in press

Walder, R. \& Folini, D. 1999b, in: Proc. 7th Intern. Conf. on Hyperbolic Problems, (Basel: Birkhäuser), in press

Willis, A.J., Schild, H., Stevens, I.R. 1995, A\&A 298, 549

\section{Discussion}

Zhekov: (1) I would like to thank Rolf for showing our (Myzsnivo, Zhekov \& Belov 1998) plot. This plot was made to demonstrate that all the nowadays available $2 \mathrm{D}$ models of radiative colliding winds are not correct. Thus, the instability seen in simulations is purely numerical. (2) The interaction of the thermal conduction with the cooling may not result in a simple picture showing a collapse of the interaction region. It might be much more complicated with appearance of secondary shocks.

Walder: (1) Well, I do not agree. I am persuaded that cold compressed layers in the interaction zone of colliding winds are indeed instable. I agree, however, that we have only poorly understood these instabilities. Let me make a comment on your results which, I think, are important. Applying a cooling cutoff is physically well founded as, at some temperature, heating by stellar and X-ray photons must balance radiative cooling. Your results may well show that the temperature at the cold compressed layer in the interaction zone of colliding winds is a sensible parameter for the non-linear outcome of the instability. (2) I completely agree that we are only at the beginning of understanding the dynamics of heat conducting shocks. This in particular for the case in which the cooling time-scale is comparable to the conduction time-scale. And this case must be considered when looking at close colliding wind binaries. 